\title{
Modified Scherrer Equation to Estimate More Accurately Nano-Crystallite Size Using XRD
}

\author{
Ahmad Monshi", Mohammad Reza Foroughi", Mohammad Reza Monshi \\ Department of Materials Engineering, Najafabad branch, Islamic Azad University, Isfahan, Iran \\ Email: ${ }^{*}$ a-monshi@cc.iut.ac.ir, "foroughi@iaun.ac.ir
}

Received July 20, 2012; revised August 20, 2012; accepted August 28, 2012

\begin{abstract}
Scherrer Equation, $L=K \lambda / \beta \cdot \cos \theta$, was developed in 1918 , to calculate the nano crystallite size $(L)$ by XRD radiation of wavelength $\lambda(\mathrm{nm})$ from measuring full width at half maximum of peaks $(\beta)$ in radian located at any $2 \theta$ in the pattern. Shape factor of $K$ can be $0.62-2.08$ and is usually taken as about 0.89 . But, if all of the peaks of a pattern are going to give a similar value of $L$, then $\beta \cdot \cos \theta$ must be identical. This means that for a typical $5 \mathrm{~nm}$ crystallite size and $\lambda C u k \alpha_{1}$ $=0.15405 \mathrm{~nm}$ the peak at $2 \theta=170^{\circ}$ must be more than ten times wide with respect to the peak at $2 \theta=10^{\circ}$, which is never observed. The purpose of modified Scherrer equation given in this paper is to provide a new approach to the kind of using Scherrer equation, so that a least squares technique can be applied to minimize the sources of errors. Modified Scherrer equation plots $\ln \beta$ against $\ln (1 / \cos \theta)$ and obtains the intercept of a least squares line regression, $\ln =K \lambda / L$, from which a single value of $L$ is obtained through all of the available peaks. This novel technique is used for a natural Hydroxyapatite (HA) of bovine bone fired at $600^{\circ} \mathrm{C}, 700^{\circ} \mathrm{C}, 900^{\circ} \mathrm{C}$ and $1100^{\circ} \mathrm{C}$ from which nano crystallite sizes of 22.8 , $35.5,37.3$ and $38.1 \mathrm{~nm}$ were respectively obtained and $900^{\circ} \mathrm{C}$ was selected for biomaterials purposes. These results show that modified Scherrer equation method is promising in nano materials applications and can distinguish between 37.3 and $38.1 \mathrm{~nm}$ by using the data from all of the available peaks.
\end{abstract}

Keywords: X-Ray Diffraction; Nano-Crystal; Scherrer Equation; Hydroxyapatite

\section{Introduction}

$\mathrm{X}$-ray diffraction is a convenient method for determining the mean size of nano crystallites in nano crystalline bulk materials. The first scientist, Paul Scherrer, published his results in a paper that included what became known as the Scherrer equation in 1981 [1].

This can be attributed to the fact that "crystallite size" is not synonymous with "particle size", while X-Ray diffraction is sensitive to the crystallite size inside the particles. From the well-known Scherrer formula the average crystallite size, $L$, is:

$$
L=\frac{K \lambda}{\beta \cdot \cos \theta}
$$

where $\lambda$ is the $\mathrm{X}$-ray wavelength in nanometer $(\mathrm{nm}), \beta$ is the peak width of the diffraction peak profile at half maximum height resulting from small crystallite size in radians and $K$ is a constant related to crystallite shape, normally taken as 0.9 . The value of $\beta$ in $2 \theta$ axis of diffraction profile must be in radians. The $\theta$ can be in degrees or radians, since the $\cos \theta$ corresponds to the same number;

${ }^{*}$ Corresponding authors.

$$
\cos \frac{\pi}{4}=\cos 45^{\circ}=\frac{\sqrt{2}}{2}
$$

It can be taken as 0.89 or 0.9 for Full Width Half Maximum (FWHM) of spherical crystals with cubic unit cells. For an excellent discussion of $K$, a good reference is the paper "Scherrer after sixty years" in 1978 [2].

In conventional approximation, the integrated width of the pure profile $(\beta)$ is separated from that of the observed diffraction profile $(B)$ assuming that both profiles are either Gaussian or Cauchy [3].

If Gaussian profile is accepted, then $B^{2}=b^{2}+\beta^{2}$ in the case of Cauchy $B=b+\beta$, where $\mathrm{b}$ is the instrument profile width. If the broadening of the pure profile is due to both crystallite size and lattice strain, one has to make another assumption concerning the shapes of the two contributing line profiles. Normally, these are supposed to be either Gaussian or both Cauchy, then $\beta=\beta_{m}^{2}+\beta_{n}^{2}$ or, $\beta=\beta_{m}+\beta_{n}$ respectively. $\beta_{m}$ is the line width resulting from small crystallite size, and $\beta_{n}$ is the line broadening due to the lattice strain. Then we have [4]:

$$
\varepsilon=\frac{\beta_{n}}{4 \cdot \operatorname{tg} \theta}
$$




$$
\text { Strain }=\text { Change in size/Original size }
$$

It is usually considered that Cauchy function is rather well approximated, while Gaussian function gave considerably larger errors. In order to separate the size and strain contribution:

$$
\varepsilon=\frac{\lambda}{L \cdot \cos \theta}+4 \varepsilon \cdot \operatorname{tg} \theta
$$

In the case of crystallite size and lattice strain, two diffraction peaks must be used to calculate two unknown parameters $L$ and $\varepsilon$.

In order to consider the Scherrer equation with obtained value of $\varepsilon$ concerning only crystallite size, when no mechanical activation such as ball milling and mechanical alloying is the case, or the crystallite size is due to a nucleation and growth at high temperatures, we must only be concerned about corrections for instrumental profile width.

The Scherrer equation predicts crystallite thickness if crystals are smaller than $1000 \AA$ or $100 \mathrm{~nm}$. The simplest way to obtain Scherrer equation is to take the derivation of Bragg's Law, $\lambda=2 d \sin \theta$.

Holding the wavelength $\lambda$ constant and allowing the diffraction angle to broaden from a sharp diffraction peak from an infinite single crystal with perfect 3-dimeintinal order. For a single crystal, the diffraction from a set of planes with the distance $d^{*}$ occurs at a precisely $\theta^{*}$, so that $\lambda=2 d^{*} \sin \theta^{*}$.

For many small nano crystals, diffraction from a lot of tiny crystals deviate $\pm \Delta \theta$ from $\theta^{*}$.

This means $2 \Delta \theta$ on the $2 \theta$ axis of diffraction pattern. The value of $\Delta \theta$ correspond to FWHM or $\beta$, which is approximately half of $2 \Delta \theta$. In other words since $\Delta \theta$ can be positive or negative, the absolute value must be taken and it reflects the half width of the shape line deviation in $2 \theta$ axis (full width at half maximum height, $\beta$ ). Derivative in $\mathrm{d}$ and $\theta$ of Bragg's Law with constant $\lambda$ gives $\lambda=$ $2 \Delta d \cdot \cos \theta \cdot \Delta \theta$.

The thickness $\Delta d=L$ can be taken as;

$$
L=\Delta d=\frac{\lambda}{2 \cos \theta \cdot \Delta \theta}=\frac{\lambda}{\cos \theta \cdot \beta}
$$

By applying a shape factor $K$, which is near the value of unit (0.9), the Scherrer equation can be given as:

$$
L=\frac{0.9 \lambda}{\beta \cdot \cos \theta}
$$

The derivation approach is taken by Alexander in Klug and Alexander "X-ray Diffraction" [3] to describe the Scherrer equation. It is also easily adoptable to describe the dependence of any two terms in the Bragg equation in terms of variability. For crystals longer than $1000 \AA$ $(100 \mathrm{~nm})$, grainy patterns can be analyzed in terms of a statistical analysis to grain size, although this is rarely done since grain size can be more easily determined from optical or electron microscopy studies in this size range.

\section{Modified Scherrer Equation}

It is assumed that if there are $N$ different peaks of a specific nano crystal in the range of $0-180^{\circ}(2 \theta)$ or 0 $90^{\circ}(\theta)$, then all of these $N$ peaks must present identical $L$ values for the crystal size. But, during the extensive research of the first author of thins paper, on different nano ceramic crystals, which were synthesized or minerally achieved, it was surprisingly observed that each peak yields a different value and there is a systematic error on the results obtained from each peak.

Further investigation approved the presence of a systematic error in Scherrer formula. In fact since

$L=K \lambda / \beta \cdot \cos \theta$, if $L$ is going to be a fixed value for different peaks of a substance, considering that $K$ and $\lambda$ and therefore $K \lambda$ are fixed values, then it is essential that $\beta \cdot \cos \theta$ be a fixed multiple during $0<2 \theta<180^{\circ}$ or $0<\theta$ $<90^{\circ}$. Suppose that for a crystallite size of $5 \mathrm{~nm}$, obtained at a peak of say $2 \theta=10^{\circ}\left(\theta=5^{\circ}\right)$ by using $K=0.89$ and $\lambda C u k \alpha_{1}=0.15405 \mathrm{~nm}$.

then $\beta_{10^{\circ}}$ must be,

$$
\beta_{10^{\circ}}=\frac{0.89(0.15405)}{5 \cos 5^{\circ}}=0.0275(\mathrm{rad})=1.576^{\circ}
$$

Now, suppose that the $N$ th peak of this nano crystal occurs at $2 \theta=170^{\circ}\left(\right.$ or $\left.\theta=85^{\circ}\right)$, then;

$$
\beta_{170^{\circ}}=\frac{0.89(0.15405)}{5 \cos 85^{\circ}}=0.3146(\mathrm{rad})=18.03^{\circ}
$$

This means that the ratio of $18.03 / 1.576=11.44$ must be applied for $\beta_{170^{\circ}} / \beta_{10^{\circ}}$. In other words if the first peak has a $\beta_{10^{\circ}}$ of around $2 \mathrm{~mm}$ on the monitor of computer plot, or for example, a paper plot $21 \mathrm{~cm}$ width on A4 paper, then the last peak must have a $\beta_{170^{\circ}}$ more than $22.88 \mathrm{~mm}$ and a base of peak more than $45.76 \mathrm{~mm}$ $(4.576 \mathrm{~cm})$.

This has never been observed and cannot be true. Modified Scherrer formula is based on the face that we must decrease the errors and obtain the average value of $L$ though all the peaks (or any number of selected peaks) by using least squares method to mathematically decrease the source of errors.

We can write the basic Scherrer formula as:

$$
\beta=\frac{K \lambda}{L \cdot \cos \theta}=\frac{K \lambda}{L} \cdot \frac{1}{\cos \theta}
$$

Now by making logarithm on both sides;

$$
\ln \beta=\ln \frac{K \lambda}{L \cdot \cos \theta}=\ln \frac{K \lambda}{L}+\ln \frac{1}{\cos \theta}
$$

If we plot the results of $\ln \beta$ against $\ln (1 / \cos \theta)$, then a straight line with a slope of around one and an intercept 
of about $\ln K / L$ must be obtained. Theoretically this straight line must be with a slope of $45^{\circ}$ since $\operatorname{tg} 45^{\circ}=1$ (Figure 1). But, since errors are associated with experimental data, the least squares method gives the best slope and most accurate $\ln K / L$. After getting the intercept, then the exponential of the intercept is obtained:

$$
e^{\ln \frac{K \lambda}{L}}=\frac{K \lambda}{L}
$$

Having $K=0.9$ and $\lambda\left(\right.$ such as $\left.\lambda C u k \alpha_{1}=0.15405 \mathrm{~nm}\right)$, a single value of $L$ in nanometer can be calculated.

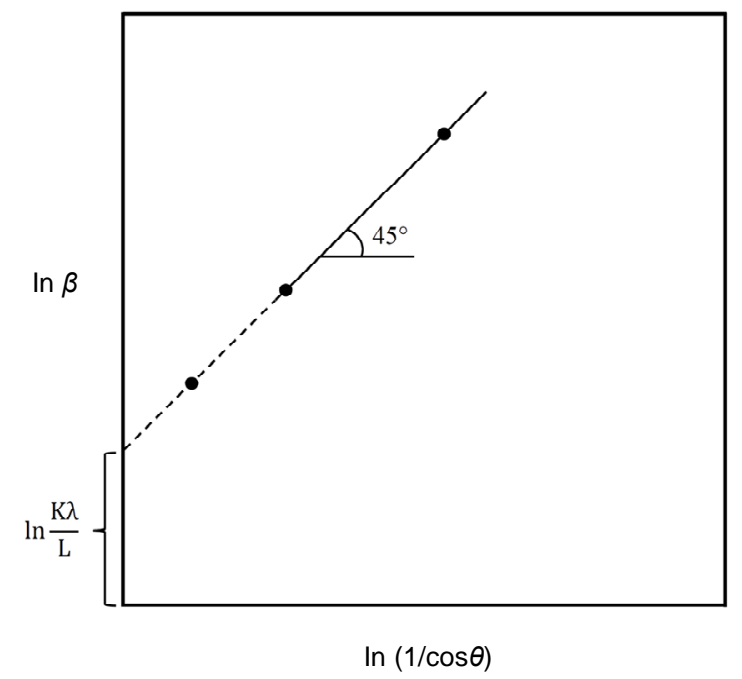

Figure 1. Modified scherrer equation plot.

\section{Experimental}

Bovine bones were boiled for $2 \mathrm{hr}$ to remove flesh and fat. The bones were heated at $60^{\circ} \mathrm{C}$ for $24 \mathrm{hr}$ to remove moisture. To prevent blackening with soot during heating, the bones are cut into small pieces of about $10 \mathrm{~mm}$ thick and heated at $400^{\circ} \mathrm{C}$ (bone ash) for $3 \mathrm{hr}$ in air to allow for evaporation of organic substances. The resulting black bone ash was heated for $2 \mathrm{hr}$ at $600^{\circ} \mathrm{C}, 700^{\circ} \mathrm{C}, 900^{\circ} \mathrm{C}$ and $1100^{\circ} \mathrm{C}[5]$.

A Philips XRD instrument with $C u k \alpha$ radiation using $40 \mathrm{KV}$ and $30 \mathrm{~mA}$, step size of $0.05^{\circ}(2 \theta)$ and scan rate of $1 \%$ min were employed. X'Pert software was used for qualitative analysis and report of $\beta$ values at FWHM at different $2 \theta$ values according to location of the peaks. According to JCPDS:9-432 standard the main peaks are from the planes of (200), (111), (002), (102), (210), (211), (112), (300), (202), (212), (310), (311), (113), (222), (312) and (213) of HA phase for $20-50$ deg. $2 \theta$.

\section{Results and Discussion}

Table 1 shows the $\beta \cdot \cos \theta$ values for different peaks at any given temperature.

The XRD patterns are observed in Figure 2 with gradual sharpness of the peaks as the soaking temperature increases, indicating the growth and increase of crystallite size.

Relatively gradual decrease in $\beta \cdot \cos \theta$ and almost increase in $L$ values $L=($ const. $/ \beta \cdot \cos \theta)$ is observed with the increase of $2 \theta$. Such as increase in crystallite

Table 1. Values of $\beta \cdot \cos \theta$ for different peaks.

\begin{tabular}{|c|c|c|c|c|c|c|}
\hline \multirow{2}{*}{$d$} & \multicolumn{5}{|c|}{ Temperature $\left({ }^{\circ} \mathrm{C}\right)$} & \multirow{2}{*}{ Calculated $L$ at $900^{\circ} \mathrm{C}(\mathrm{nm})$} \\
\hline & 400 & 600 & 700 & 900 & 1100 & \\
\hline$(200)$ & - & - & $5.06 \times 10^{-3}$ & $5.06 \times 10^{-3}$ & $5.06 \times 10^{-3}$ & 27.1 \\
\hline$(111)$ & - & - & - & $5.05 \times 10^{-3}$ & $5.05 \times 10^{-3}$ & 27.1 \\
\hline$(002)$ & $2.24 \times 10^{-3}$ & $5.02 \times 10^{-3}$ & $3.35 \times 10^{-3}$ & $3.35 \times 10^{-3}$ & $3.35 \times 10^{-3}$ & 40.9 \\
\hline$(102)$ & - & - & $2.50 \times 10^{-3}$ & $3.34 \times 10^{-3}$ & $2.50 \times 10^{-3}$ & 41 \\
\hline$(210)$ & - & - & - & $3.23 \times 10^{-3}$ & $2.50 \times 10^{-3}$ & 42.4 \\
\hline$(211)$ & $9.90 \times 10^{-3}$ & $11.5 \times 10^{-3}$ & $2.48 \times 10^{-3}$ & $3.31 \times 10^{-3}$ & $2.48 \times 10^{-3}$ & 41.4 \\
\hline$(112)$ & - & - & $2.48 \times 10^{-3}$ & $2.48 \times 10^{-3}$ & $2.48 \times 10^{-3}$ & 55.2 \\
\hline$(300)$ & - & - & $2.47 \times 10^{-3}$ & $3.30 \times 10^{-3}$ & $3.30 \times 10^{-3}$ & 41.5 \\
\hline (202) & - & - & $3.29 \times 10^{-3}$ & $3.29 \times 10^{-3}$ & $3.29 \times 10^{-3}$ & 41.6 \\
\hline$(310)$ & $11.3 \times 10^{-3}$ & $12.9 \times 10^{-3}$ & $2.42 \times 10^{-3}$ & $2.16 \times 10^{-3}$ & $3.23 \times 10^{-3}$ & 63.4 \\
\hline (311) & - & - & $2.41 \times 10^{-3}$ & $4.81 \times 10^{-3}$ & $2.41 \times 10^{-3}$ & 28.5 \\
\hline (113) & - & - & $3.19 \times 10^{-3}$ & $6.38 \times 10^{-3}$ & $3.19 \times 10^{-3}$ & 21.5 \\
\hline$(222)$ & $7.88 \times 10^{-3}$ & $7.88 \times 10^{-3}$ & $3.16 \times 10^{-3}$ & $2.11 \times 10^{-3}$ & $3.16 \times 10^{-3}$ & 65 \\
\hline (312) & - & - & $3.14 \times 10^{-3}$ & $3.14 \times 10^{-3}$ & $3.14 \times 10^{-3}$ & 43.6 \\
\hline (213) & $9.35 \times 10^{-3}$ & $6.25 \times 10^{-3}$ & $3.89 \times 10^{-3}$ & $3.12 \times 10^{-3}$ & $2.09 \times 10^{-3}$ & 44 \\
\hline
\end{tabular}




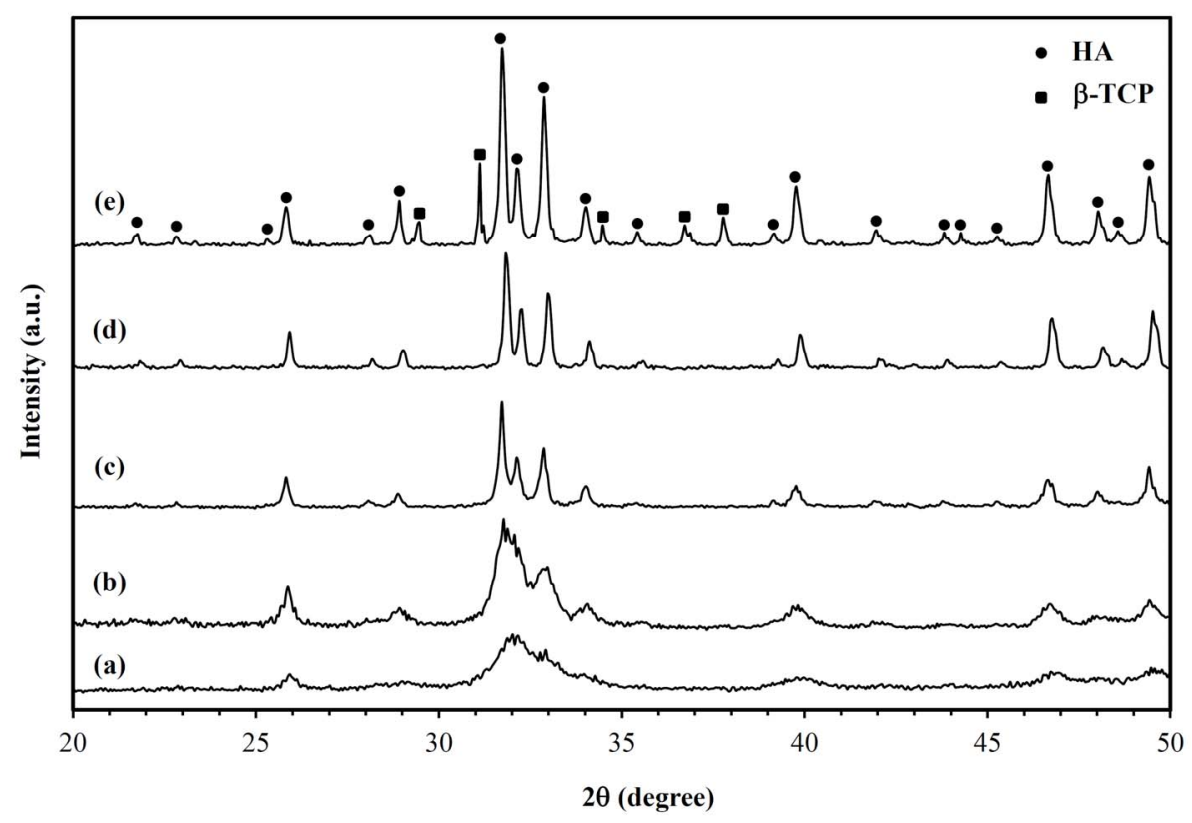

Figure 2. Patterns of XRD analysis related to natural HA of thermal analysis at temperatures of: (a) $400^{\circ} \mathrm{C}$ (bone ash); (b) $600^{\circ} \mathrm{C}$; (c) $700^{\circ} \mathrm{C}$; (d) $900^{\circ} \mathrm{C}$ and (e) $1100^{\circ} \mathrm{C}$.

size of HA were also observed by Shipmen et al. [6]. The samples fired at $600^{\circ} \mathrm{C}-1100^{\circ} \mathrm{C}$ were used.

Figure 3 indicates four plots of $\ln \beta$ vs. $\ln (1 \cos \theta)$ for individual soaking temperatures; together with the equations of linear least squares method obtained from linear regression of data in excel plots. Due to sources of error in measuring $\beta$ and treating for different available peaks, since the $\beta \cdot \cos \theta$ multiple is not really a constant value for all the peaks, $\sigma \lambda o \pi \varepsilon \sigma$ deviate from $45^{\circ}$ and are negative in some cases. The reason that the slopes are negative are due to the fact that at higher angles, of $2 \theta$, with lower values of $\cos \theta$ and higher values of $\ln (1 \cos \theta)$, the amounts of $\beta$ observed and measured are less than it must be according to Scherrer's formula. This means that a $45^{\circ}$ slope for the linear plots are hardly achieved. The errors involved in Scherrer equation when employing different peaks are the main cause of scattering of the points. Some other sources of error are measuring $\ln \beta$ and $\ln (1 \cos \theta)$. The significance of this work is to minimize the errors by applying a method to use least squares technique for obtaining the best results.

The modified Scherrer equation can provide the advantage of decreasing the sum of absolute values of errors, $\quad \sum( \pm \Delta \ln \beta)^{2}$, and producing a single line through the points to give a single value of intercept $\ln K / L$. At $600^{\circ} \mathrm{C}$, the linear regression plot is obtained as $y=4.8149 x-5.1196$. This is equivalent to $\ln \beta=4.8149(\ln 1 / \cos \theta)+\ln K \lambda / L$. From this line, the intercept is -5.1196 and $K \lambda / L=e^{-5.1196}=0.006$ and

$$
L=(0.89)(0.15405) / 0.006=22.8(\mathrm{~nm})
$$

It is interesting to notice that although variations exist in $\ln \beta$ values, but the intercept systematically changes as $-5.1196,-5.5542,-5.6054$ and -5.6276 respectively for $600^{\circ} \mathrm{C}, 700^{\circ} \mathrm{C}, 900^{\circ} \mathrm{C}$ and $1100^{\circ} \mathrm{C}$. The treatment is shown in Table 2 leading to values of 22.8, 35.5, 37.3 and 38.1 nanometers respectively. Danilchenko et al. [6] have also reported the bone mineral crystalline size of about $20 \mathrm{~nm}$.

The plot of crystal size of HA vs. firing temperature is given in Figure 4. From this figure it can be understood that the shape is similar to that of parabolic Law. Crystallinity sharply increase from $600^{\circ} \mathrm{C}$ to $700^{\circ} \mathrm{C}$, but the rate slows down from $700^{\circ} \mathrm{C}$ to $1100^{\circ} \mathrm{C}$. It seems that the driving force for the growth of nano crystallite size of HA is highly provided when the temperature increases from $600^{\circ} \mathrm{C}$ to $700^{\circ} \mathrm{C}$, but is less affected by higher temperature increases. In other words the experimental activation energy for the growth of nano HA crystallites can be provided in $600^{\circ} \mathrm{C}$ to $700^{\circ} \mathrm{C}$. The values of $\Delta L / \Delta T$ representing the rate of growth in size are plotted against Temperature $\left({ }^{\circ} \mathrm{C}\right)$ in Figure 5. This figure can confirm

Table 2. Treatment of linear plots to obtain nano size of crystallites.

\begin{tabular}{ccc}
\hline Temperature $\left({ }^{\circ} \mathrm{C}\right)$ & $e^{\ln \frac{k \lambda}{L}}=\frac{0.89 \times 0.154051}{L}$ & $\boldsymbol{L} \mathbf{( n m )}$ \\
\hline 600 & $e^{-5.1196}=0.006$ & 22.8 \\
700 & $e^{-5.5542}=0.00387$ & 35.5 \\
900 & $e^{-5.6054}=0.00368$ & 37.3 \\
1100 & $e^{-5.16276}=0.0036$ & 38.1 \\
\hline
\end{tabular}



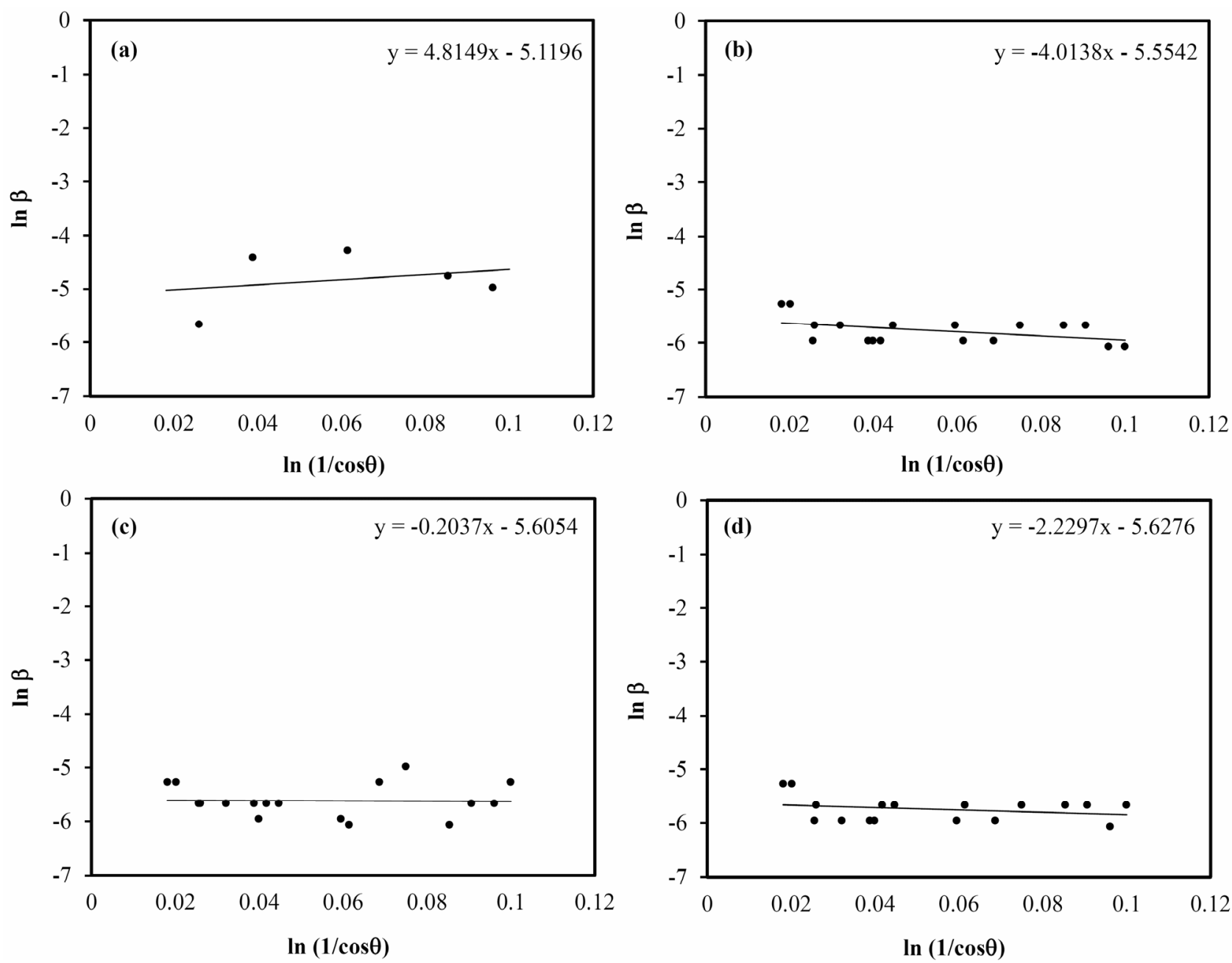

Figure 3. Linear plots of modified scherrer equation and obtained intercepts for different firings of ha.

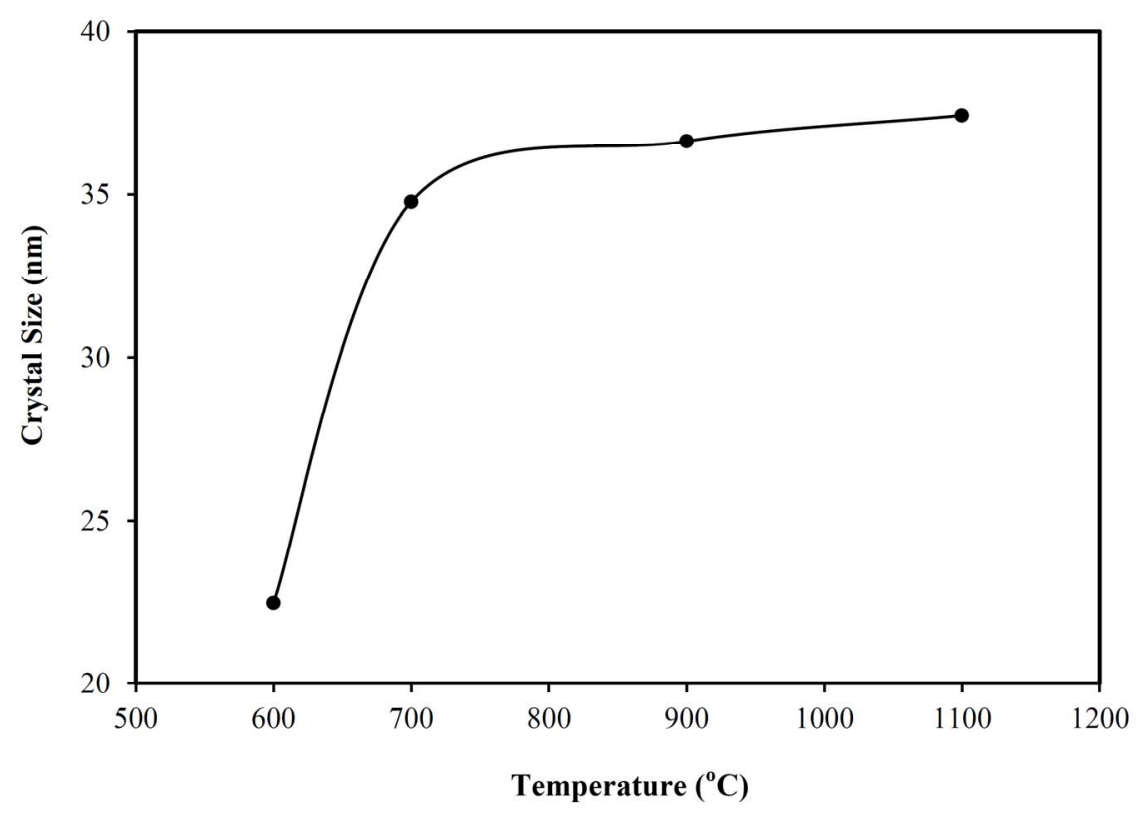

Figure 4. Crystal size (nm) of HA against firing temperature. 


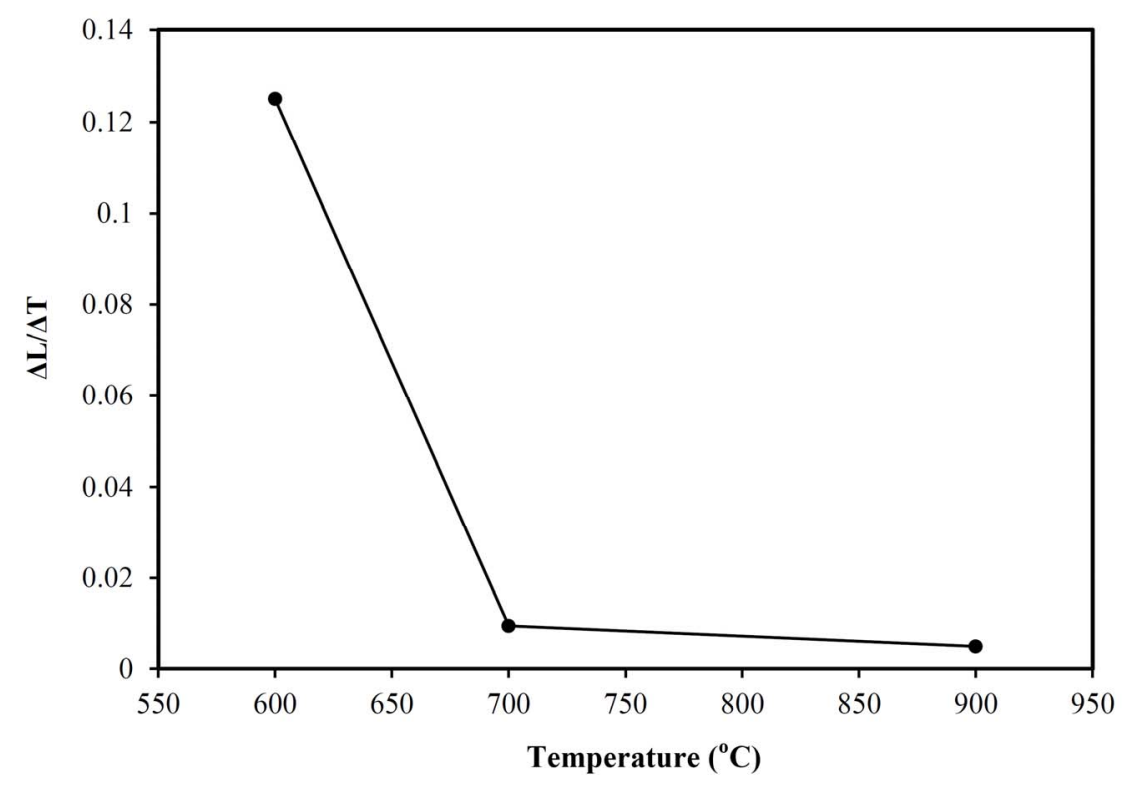

Figure 5. Plot of $\Delta L / \Delta T$ (slope of plot in Figure 4) against temperature ( ${ }^{\circ} \mathrm{C}$ ).

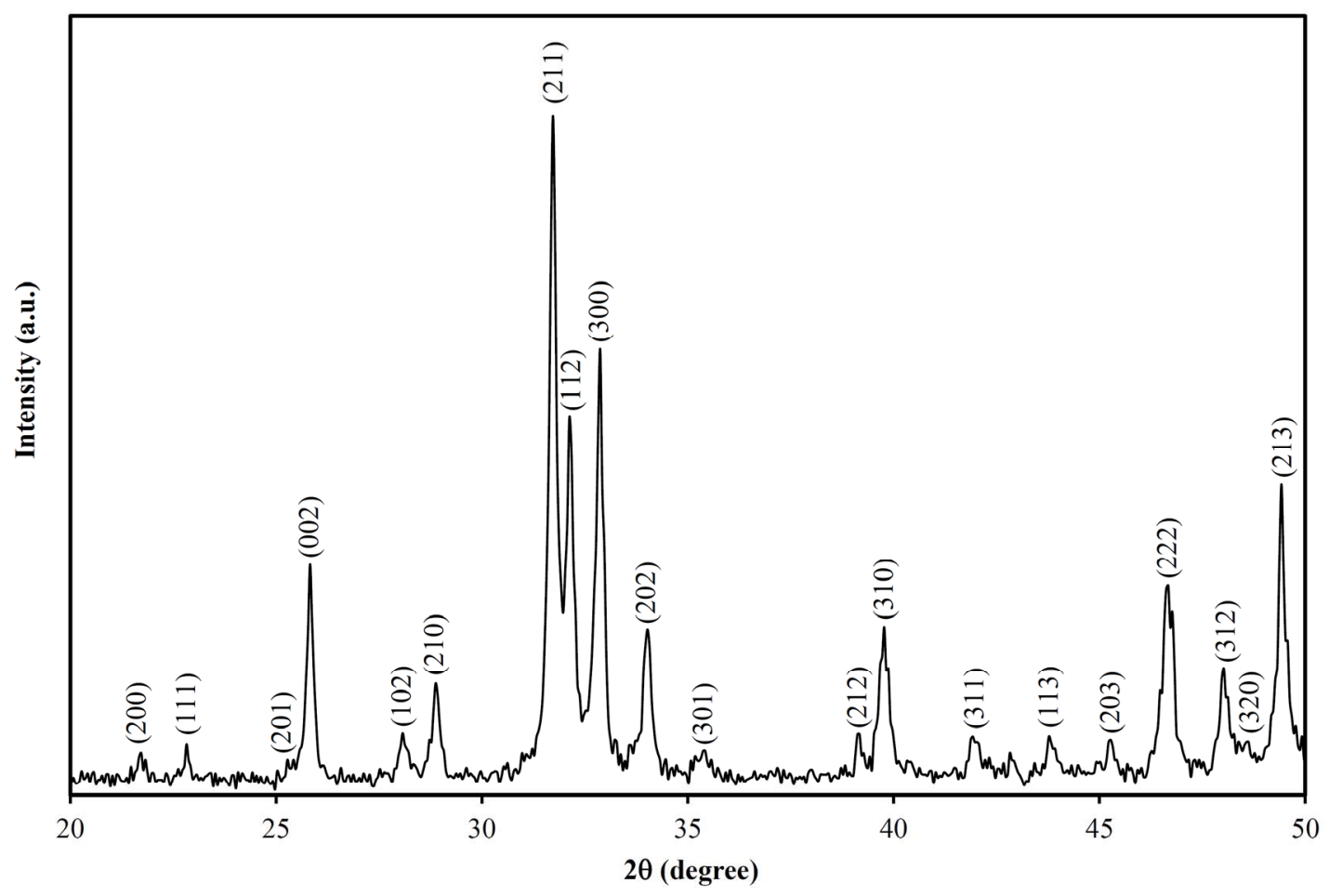

Figure 6. All of the diffraction planes of $\mathrm{HA}$ firing at $900^{\circ} \mathrm{C}$.

the about discussion that the most increase in size of nano $\mathrm{HA}$ or $\Delta L / \Delta T$ occurs at $600^{\circ} \mathrm{C}$ to $700^{\circ} \mathrm{C}$.

Some peaks of $\beta$-Tricalcium phosphate $(\beta$-TCP) shows up at $1100^{\circ} \mathrm{C}$. In order to obtain well developed crystallines of HA without the side effect of $\beta$-TCP, firing at $900^{\circ} \mathrm{C}$ was selected for biomaterial purposes. The XRD pattern of this sample is shown in Figure 6, showing all of the diffraction planes.

\section{Conclusions}

1) Scherrer equation systematically show increased values of nano crystalline size as $d$ values decrease and $2 \theta$ values increase, since $\beta \cdot \cos \theta$ cannot be maintained as constant.

2) If $\ln \beta$ is plotted against $\ln (1 / \cos \theta)$ and least squares method is employed, the intercept gives $\ln =K \lambda / L$, from 
which a single value of $L$ can be obtained.

3) Modified Scherrer equation can provide the advantage of decreasing the errors or $\sum( \pm \Delta \ln \beta)^{2}$ to give a more accurate value of $L$ from all or some of the different peaks.

4) In a study on natural hydroxyapatite of bovine bone, fired at $600^{\circ} \mathrm{C}, 700^{\circ} \mathrm{C}, 90^{\circ} \mathrm{C}$ and $1100^{\circ} \mathrm{C}$, the values of $22.8,35.5,37.3$ and $38.1 \mathrm{~nm}$ were respectively obtained for nano crystallite size.

5) Since at $1100^{\circ} \mathrm{C}$, peaks of $\beta$-TCP start to show up, $900^{\circ} \mathrm{C}$ firing was selected for producing HA for biomaterials purposes.

\section{REFERENCES}

[1] P. Scherrer, "Bestimmung der Grösse und der Inneren Struktur von Kolloidteilchen Mittels Röntgenstrahlen, Nachrichten von der Gesellschaft der Wissenschaften, Göttingen," Mathematisch-Physikalische Klasse, Vol. 2, 1918, pp. 98-100.

[2] J. Langford and A. Wilson, "Scherrer after Sixty Years: A Survey and Some New Results in the Determination of
Crystallite Size," Journal of Applied Crystallography, Vol. 11, 1978, pp. 102-103. doi:10.1107/S0021889878012844

[3] H. Klug and L. Alexander, "X-Ray Diffraction Procedure for Polycrystallite and Amorphous Materials," 2nd Edition, John Wiley and Sons, New York, 1974.

[4] S. Dinilchenko, O. Kukharenko, C. Moseke, I. Protsenko, L. Sukhodub and B. Sulkio-Cleff, "Determination of the Bone Mineral Crystallite Size and Lattice Strain from Diffraction Line Broadening," Research and Technology Crystal, Vol. 37, No. 11, 2002, pp. 1234-1240.

[5] M. R. Foroughi, S. Karbasi and R. Ebrahimi-Kahrizsangi, "Physical and Mechanical Properties of a Poly-3-Hydroxylbutyrate-coated Nanocrystalline Hydroxyapatite Scaffold for Bone Tissue Engineering," Journal of Porous Materials, Vol. 19, No. 5, 2011, pp. 667-675. doi:10.1007/s10934-011-9518-1.

[6] P. Shipman, G. Foster and M. Schoeninger, "Burnt Bones and Teeth: An Experimental Study of Color, Morphology, Crystal Structure and Shrinkage," Journal of Archaeological Science, Vol. 11, No. 4, 1984, pp. 307-325. doi:10.1016/0305-4403(84)90013-X 